\title{
Frage zur Beurteilung von Fischfrikadellen/Fischburgern bezüglich zu erwartender Beschaffenheit und Kennzeichnung
}

\author{
Thomas Reiche
}

(C) Bundesamt für Verbraucherschutz und Lebensmittelsicherheit (BVL) 2011

Beim Zentralen Institut des Sanitätsdienstes der Bundeswehr Koblenz, Laborabteilung II, Veterinärmedizin wurden 2009 die Fischerzeugnisse Fischburger und Fischfrikadellen der Firma xxxx GmbH zur Untersuchung vorgelegt. Hierbei wurden folgende Untersuchungsergebnisse gewonnen:

\section{Deklaration}

1. Verkehrsbezeichnung: Fischburger

Zutatenliste: Fisch (Alaska-Seelachs, Seelachs, Seehecht, Rotbarsch) (50\%), Weizenmehl, Wasser, Kartoffeln, pflanzliches Öl, Zwiebeln, Speisesalz, Porree, Stabilisator E450, Emulgator E471, Antioxidationsmittel E223, Kräuter, Gewürze, Milchzucker, Hefe, Gewürzextrakt

2. Verkehrsbezeichnung: Fischfrikadelle, paniert, vorgebraten

Zutatenliste: Zutaten: Fisch (Alaska-Seelachs, Seelachs, Seehecht, Hold, Kabeljau, Pangasius) (55\%), Wasser, Weizenmehl, Kartoffeln, pflanzliches Öl, Porree, Speisesalz, Kräuter, Zwiebeln, Milchzucker, Gewürze, Stärke, natürliches Aroma

\section{Vergleichende Prüfung}

In den einfach beschreibenden sensorischen Prüfungen der o. a. Produkte wurde festgestellt, dass die
Produkte im nicht zubereiteten, aufgetauten Zustand nach Entfernung der Panade ausschließlich aus einem streichfähigen, grauweißen, mehligen Teig mit einzelnen, gerade noch erkennbaren, maximal 0,3 cm Durchmesser großen grauweißen Fischmuskelstücken bestanden.

In den gravimetrischen Prüfungen wurden die als Fischmuskelfleisch erkennbaren Partikel/Stückchen präpariert und der Gewichtsanteil am Gesamtprodukt bestimmt. Es wurden in den geprüften Proben zwischen 1,8\% und 16,5\% Fischmuskelfleischstücke (im Mittel 10,7 \%) nachgewiesen.

\section{Vorgaben Leitsätze für Fische, Krebs- und Weichtiere und Erzeugnisse daraus}

Nach Kapitel II B Ziffer 2 b werden Fischfrikadellen und Fischburger und andere geformte Erzeugnisse aus Fischfleisch aus Blöcken von praktisch grätenfreien Fischfilets, von anderem zusammenhängendem Fischfleisch und von zerkleinertem Fischfleisch auch mit Zusatz von Bindemitteln sowie würzenden Zutaten (auch mit Beigaben anderer Lebensmittel), in der Regel paniert oder in Backteig hergestellt. Nach Ziffer $4 \mathrm{~d}$ hat bei Fischerzeugnissen aus zerkleinertem Fischfleisch der Fischanteil mindestens $50 \%$ zu betragen. Unter dem Begriff „,zerkleinertes Fischfleisch“ ist im Sinne Kapitel I A Ziffer 4 i sowohl Hack, als auch Farce, Mus ohne Haut und Gräten, jedoch mit histologisch nachweisbarer Faserstruktur zu verstehen.
T. Reiche

Zentrales Institut der Bundeswehr, Mainz, Germany 